Proyecciones Journal of Mathematics

Vol. 34, No 4, pp. 401-411, December 2015.

Universidad Católica del Norte

Antofagasta - Chile

\title{
A New Closed Graph Theorem on Product Spaces
}

\author{
S. Zhong \\ Tianjin University, China \\ and \\ G. Zhao \\ Weixian Middle School, China \\ Received : July 2015. Accepted : September 2015
}

\begin{abstract}
We obtain a new version of closed graph theorem on product spaces. Fernandez's closed graph theorem for bilinear and multilinear mappings follows as a special case.
\end{abstract}

Subjclass [2010] : Primary 46A30; Secondary 47 H99.

Keywords : Closed graph theorem, product spaces, bilinear mappings, bi-mappings, multi-mappings. 


\section{Introduction}

The classical closed graph theorem [1] says that, if $X, Y$ are Banach spaces (or Fréchet spaces) and $f: X \rightarrow Y$ a linear mapping with closed graph, then $f$ is continuous. As the closed graph theorem is a famous theorem, there have been a lot of results on it. Especially, we can find many new types of closed graph theorem recently, such as $[2,5,6,7,8,10,11,12]$.

But whether the closed graph theorem holds for mappings defined on product spaces?

In the first years, people considered the bilinear mappings defined on product spaces. P. J. Cohen [3], in 1974, gave us a negative answer for an equivalent version of the above classical closed graph theorem. However, in 1996, C. S. Fernandez [4] showed the above classical closed graph theorem holds for bilinear and multilinear mappings defined on product spaces.

In this paper, we will give another version of closed graph theorem for bimappings and multi-mappings on product spaces, and show that the family of bilinear (or multilinear) mappings with closed graph is just a subfamily of bi-mappings (or multi-mappings) with closed graph. Especially, from our results, the version of closed graph theorem in $[11,12]$ can easily be obtained and the closed graph theorem in [4] is just a special case.

\section{Main results}

Definition 2.1. Let $X_{1}, X_{2}$ and $Y$ be topological vector spaces. A mapping $f: X_{1} \times X_{2} \rightarrow Y$ is said to be a bi-mapping if for each $x^{1}, x_{n}^{1}, u_{n}^{1} \in X_{1}$ and $x^{2}, x_{n}^{2}, u_{n}^{2} \in X_{2}$ with $n \in \mathbf{N}$ the following (1), (2) and (3) hold:

(1) if $f\left(x_{n}^{1}, 0\right) \rightarrow 0$ and $f\left(u_{n}^{1}, 0\right) \rightarrow 0$, then $f\left(x_{n}^{1}+u_{n}^{1}, 0\right) \rightarrow 0$;

if $f\left(0, x_{n}^{2}\right) \rightarrow 0$ and $f\left(0, u_{n}^{2}\right) \rightarrow 0$, then $f\left(0, x_{n}^{2}+u_{n}^{2}\right) \rightarrow 0$;

(2) if $f\left(x_{n}^{1}-x^{1}, 0\right) \rightarrow 0$ and $t_{n} \rightarrow t$ in the scalar field $\mathbf{K}$, then

$$
f\left(t_{n} x_{n}^{1}-t x^{1}, 0\right) \rightarrow 0
$$

if $f\left(0, x_{n}^{2}-x^{2}\right) \rightarrow 0$ and $t_{n} \rightarrow t$ in the scalar field $\mathbf{K}$, then

$$
f\left(0, t_{n} x_{n}^{2}-t x^{2}\right) \rightarrow 0
$$

(3) if $x_{n}^{1} \rightarrow x^{1}$ and $x_{n}^{2} \rightarrow x^{2}$, then

$$
f\left(x_{n}^{1}, x_{n}^{2}\right) \rightarrow f\left(x^{1}, x^{2}\right)
$$


if and only if

$$
f\left(x_{n}^{1}-x^{1}, 0\right) \rightarrow 0 \text { and } f\left(0, x_{n}^{2}-x^{2}\right) \rightarrow 0 .
$$

Note that a Fréchet space is a complete metrizable linear space. However, a Fréchet space is also a separated complete paranormed space [9].

Theorem 2.2. Let $X_{1}, X_{2}$ and $Y$ be Fréchet spaces. If $f: X_{1} \times X_{2} \rightarrow Y$ is a bi-mapping with closed graph, then $f$ is continuous.

Proof. Let $X_{1}=\left(X_{1},\|\cdot\|_{1}\right), X_{2}=\left(X_{2},\|\cdot\|_{2}\right)$ and $Y=(Y,\|\cdot\|)$ where $\|\cdot\|_{1},\|\cdot\|_{2}$ and $\|\cdot\|$ are paranorms $[9]$ on $X_{1}, X_{2}$ and $Y$ separatively. Define a mapping

$$
d:\left(X_{1} \times X_{2}\right) \times\left(X_{1} \times X_{2}\right) \rightarrow \mathbf{R}
$$

by

$$
d\left(\left(x_{1}, x_{2}\right),\left(u_{1}, u_{2}\right)\right)=\left\|x_{1}-u_{1}\right\|_{1}+\left\|x_{2}-u_{2}\right\|_{2}+\left\|f\left(x_{1}, x_{2}\right)-f\left(u_{1}, u_{2}\right)\right\|
$$

for all $x_{1}, u_{1} \in X_{1}$ and $x_{2}, u_{2} \in X_{2}$. It is easy to know $d$ is a metric on $X_{1} \times X_{2}$.

Let $\left\{\left(x_{n}^{1}, x_{n}^{2}\right)\right\}$ be Cauchy in $\left(X_{1} \times X_{2}, d\right)$. Then

$d\left(\left(x_{n}^{1}, x_{n}^{2}\right),\left(x_{m}^{1}, x_{m}^{2}\right)\right)=\left\|x_{n}^{1}-x_{m}^{1}\right\|_{1}+\left\|x_{n}^{2}-x_{m}^{2}\right\|_{2}+\left\|f\left(x_{n}^{1}, x_{n}^{2}\right)-f\left(x_{m}^{1}, x_{m}^{2}\right)\right\| \rightarrow 0$

when $n, m \rightarrow+\infty$. So $\left\{x_{n}^{1}\right\},\left\{x_{n}^{2}\right\}$ and $\left\{f\left(x_{n}^{1}, x_{n}^{2}\right)\right\}$ are Cauchy in $\left(X_{1},\|\cdot\|_{1}\right)$, $\left(X_{2},\|\cdot\|_{2}\right)$ and $(Y,\|\cdot\|)$ respectively. Since $X_{1}, X_{2}$ and $Y$ are complete, there exist $x^{1} \in X_{1}, x^{2} \in X_{2}$ and $y \in Y$ such that

$$
\left\|x_{n}^{1}-x^{1}\right\|_{1} \rightarrow 0, \quad\left\|x_{n}^{2}-x^{2}\right\|_{2} \rightarrow 0, \quad\left\|f\left(x_{n}^{1}, x_{n}^{2}\right)-y\right\| \rightarrow 0 .
$$

But $f$ has closed graph. Then $y=f\left(x^{1}, x^{2}\right)$ and

$$
\begin{gathered}
d\left(\left(x_{n}^{1}, x_{n}^{2}\right),\left(x^{1}, x^{2}\right)\right)=\left\|x_{n}^{1}-x^{1}\right\|_{1}+\left\|x_{n}^{2}-x^{2}\right\|_{2}+\left\|f\left(x_{n}^{1}, x_{n}^{2}\right)-f\left(x^{1}, x^{2}\right)\right\| \\
=\left\|x_{n}^{1}-x^{1}\right\|_{1}+\left\|x_{n}^{2}-x^{2}\right\|_{2}+\left\|f\left(x_{n}^{1}, x_{n}^{2}\right)-y\right\| \rightarrow 0
\end{gathered}
$$

when $n \rightarrow+\infty$. Hence, $\left(X_{1} \times X_{2}, d\right)$ is a complete metric space.

Let $\left(x_{n}^{1}, x_{n}^{2}\right) \rightarrow\left(x^{1}, x^{2}\right)$ and $\left(u_{n}^{1}, u_{n}^{2}\right) \rightarrow\left(u^{1}, u^{2}\right)$ in $\left(X_{1} \times X_{2}, d\right)$. Then

$d\left(\left(x_{n}^{1}, x_{n}^{2}\right),\left(x^{1}, x^{2}\right)\right)=\left\|x_{n}^{1}-x^{1}\right\|_{1}+\left\|x_{n}^{2}-x^{2}\right\|_{2}+\left\|f\left(x_{n}^{1}, x_{n}^{2}\right)-f\left(x^{1}, x^{2}\right)\right\| \rightarrow 0$,

$d\left(\left(u_{n}^{1}, u_{n}^{2}\right),\left(u^{1}, u^{2}\right)\right)=\left\|u_{n}^{1}-u^{1}\right\|_{1}+\left\|u_{n}^{2}-u^{2}\right\|_{2}+\left\|f\left(u_{n}^{1}, u_{n}^{2}\right)-f\left(u^{1}, u^{2}\right)\right\| \rightarrow 0$ 
when $n \rightarrow+\infty$. As $f$ is a bi-mapping, by (3),

$$
\left\|f\left(x_{n}^{1}-x^{1}, 0\right)\right\| \rightarrow 0, \quad\left\|f\left(0, x_{n}^{2}-x^{2}\right)\right\| \rightarrow 0,
$$

and

$$
\left\|f\left(u_{n}^{1}-u^{1}, 0\right)\right\| \rightarrow 0, \quad\left\|f\left(0, u_{n}^{2}-u^{2}\right)\right\| \rightarrow 0 .
$$

And by (1),

$$
\left\|f\left(x_{n}^{1}+u_{n}^{1}-x^{1}-u^{1}, 0\right)\right\| \rightarrow 0, \quad\left\|f\left(0, x_{n}^{2}+u_{n}^{2}-x^{2}-u^{2}\right)\right\| \rightarrow 0 .
$$

Since

$$
\left\|x_{n}^{1}+u_{n}^{1}-x^{1}-u^{1}\right\|_{1} \leq\left\|x_{n}^{1}-x^{1}\right\|_{1}+\left\|u_{n}^{1}-u^{1}\right\|_{1} \rightarrow 0
$$

and

$$
\left\|x_{n}^{2}+u_{n}^{2}-x^{2}-u^{2}\right\|_{2} \leq\left\|x_{n}^{2}-x^{2}\right\|_{2}+\left\|u_{n}^{2}-u^{2}\right\|_{2} \rightarrow 0,
$$

by (3) again,

$$
\left\|f\left(x_{n}^{1}+u_{n}^{1}, x_{n}^{2}+u_{n}^{2}\right)-f\left(x^{1}+u^{1}, x^{2}+u^{2}\right)\right\| \rightarrow 0 .
$$

Thus,

$$
\begin{gathered}
d\left(\left(x_{n}^{1}+u_{n}^{1}, x_{n}^{2}+u_{n}^{2}\right),\left(x^{1}+u^{1}, x^{2}+u^{2}\right)\right) \\
=\left\|x_{n}^{1}+u_{n}^{1}-x^{1}-u^{1}\right\|_{1}+\left\|x_{n}^{2}+u_{n}^{2}-x^{2}-u^{2}\right\|_{2} \\
+\left\|f\left(x_{n}^{1}+u_{n}^{1}, x_{n}^{2}+u_{n}^{2}\right)-f\left(x^{1}+u^{1}, x^{2}+u^{2}\right)\right\| \rightarrow 0
\end{gathered}
$$

so the additive operation is continuous on $\left(X_{1} \times X_{2}, d\right)$.

Let $\left(x_{n}^{1}, x_{n}^{2}\right) \rightarrow\left(x^{1}, x^{2}\right)$ in $\left(X_{1} \times X_{2}, d\right)$ and $t_{n} \rightarrow t$ in the scalar field $\mathbf{K}$. Then

$d\left(\left(x_{n}^{1}, x_{n}^{2}\right),\left(x^{1}, x^{2}\right)\right)=\left\|x_{n}^{1}-x^{1}\right\|_{1}+\left\|x_{n}^{2}-x^{2}\right\|_{2}+\left\|f\left(x_{n}^{1}, x_{n}^{2}\right)-f\left(x^{1}, x^{2}\right)\right\| \rightarrow 0$

so

$$
\left\|x_{n}^{1}-x^{1}\right\|_{1} \rightarrow 0, \quad\left\|x_{n}^{2}-x^{2}\right\|_{2} \rightarrow 0, \quad\left\|f\left(x_{n}^{1}, x_{n}^{2}\right)-f\left(x^{1}, x^{2}\right)\right\| \rightarrow 0 .
$$

By (3), $\left\|f\left(x_{n}^{1}-x^{1}, 0\right)\right\| \rightarrow 0$ and $\left\|f\left(0, x_{n}^{2}-x^{2}\right)\right\| \rightarrow 0$. And by $(2)$,

$$
\left\|f\left(t_{n} x_{n}^{1}-t x^{1}, 0\right)\right\| \rightarrow 0, \quad\left\|f\left(0, t_{n} x_{n}^{2}-t x^{2}\right)\right\| \rightarrow 0 .
$$


By (3) again,

$$
f\left(t_{n} x_{n}^{1}, t_{n} x_{n}^{2}\right) \rightarrow f\left(t x^{1}, t x^{2}\right)
$$

since $\left\|t_{n} x_{n}^{1}-t x^{1}\right\|_{1} \rightarrow 0$ and $\left\|t_{n} x_{n}^{2}-t x^{2}\right\|_{2} \rightarrow 0$. Hence,

$$
\begin{gathered}
d\left(t_{n}\left(x_{n}^{1}, x_{n}^{2}\right), t\left(x^{1}, x^{2}\right)\right)=\left\|t_{n} x_{n}^{1}-t x^{1}\right\|_{1}+\left\|t_{n} x_{n}^{2}-t x^{2}\right\|_{2} \\
+\left\|f\left(t_{n} x_{n}^{1}, t_{n} x_{n}^{2}\right)-f\left(t x^{1}, t x^{2}\right)\right\| \rightarrow 0
\end{gathered}
$$

so the scalar multiplication is also continuous in $\left(X_{1} \times X_{2}, d\right)$.

It follows that $\left(X_{1} \times X_{2}, d\right)$ is a complete metric vector space. Namely, it is a Fréchet space. Let $I\left(x_{1}, x_{2}\right)=\left(x_{1}, x_{2}\right)$ for each $\left(x_{1}, x_{2}\right) \in X_{1} \times X_{2}$. Then

$$
I:\left(X_{1} \times X_{2}, d\right) \rightarrow X_{1} \times X_{2}
$$

is continuous, one to one and surjective. By Banach open mapping theorem [9], the inverse

$$
I^{-1}: X_{1} \times X_{2} \rightarrow\left(X_{1} \times X_{2}, d\right)
$$

is continuous too.

Let $\left\|x_{n}^{1}-x^{1}\right\|_{1} \rightarrow 0$ and $\left\|x_{n}^{2}-x^{2}\right\|_{2} \rightarrow 0$.

Then

$$
\left(x_{n}^{1}, x_{n}^{2}\right)=I^{-1}\left(x_{n}^{1}, x_{n}^{2}\right) \rightarrow I^{-1}\left(x^{1}, x^{2}\right)=\left(x^{1}, x^{2}\right)
$$

in $\left(X_{1} \times X_{2}, d\right)$ so

$d\left(\left(x_{n}^{1}, x_{n}^{2}\right),\left(x^{1}, x^{2}\right)\right)=\left\|x_{n}^{1}-x^{1}\right\|_{1}+\left\|x_{n}^{2}-x^{2}\right\|_{2}+\left\|f\left(x_{n}^{1}, x_{n}^{2}\right)-f\left(x^{1}, x^{2}\right)\right\| \rightarrow 0$.

Hence, $\left\|f\left(x_{n}^{1}, x_{n}^{2}\right)-f\left(x^{1}, x^{2}\right)\right\| \rightarrow 0$ so $f\left(x_{n}^{1}, x_{n}^{2}\right) \rightarrow f\left(x^{1}, x^{2}\right)$ in $Y$.

Remark 2.3. If, in Theorem 2.2, $X_{1}, X_{2}, Y$ are Banach spaces, then every bilinear mapping $f$ from $X_{1} \times X_{2}$ to $Y$ with closed graph is a bi-mapping with closed graph. So the closed graph theorem for bilinear mappings [4] is just a special case of Theorem 2.2.

Remark 2.4. For a weakly quasi-linear mapping $f$ [12] from a Hausdorff topological vector space $X$ to a topological vector space $Y$, define $g: X \times$ $Z \rightarrow Y$ by $g(x, z)=f(x)$ where $Z$ is a topological vector space. Then $g$ is a bi-mapping from $X \times Z$ to $Y$, even $g$ is with closed graph when $f$ is with closed graph. Hence, the result of closed graph theorem in [11, 12] can immediately be obtained from Theorem 2.2. 
However, there are some bi-mappings which are not bilinear, not weakly quasi-linear, and even continuous.

Example 2.5. Define $f: \mathbf{R}^{2} \rightarrow \mathbf{R}$ by $f(x, u)=x^{2} u^{2}$.

It is obvious that $f$ satisfies the condition (1), (2) and the necessity of (3) in Definition 2.1. Let

$$
x_{n} \rightarrow x, \quad u_{n} \rightarrow u, \quad f\left(x_{n}-x, 0\right) \rightarrow 0, \quad f\left(0, u_{n}-u\right) \rightarrow 0 .
$$
So

Then $x_{n}^{2} u_{n}^{2} \rightarrow x^{2} u^{2}$ in real space $\mathbf{R}$ by the property of product limit.

$$
f\left(x_{n}, u_{n}\right)=x_{n}^{2} u_{n}^{2} \rightarrow x^{2} u^{2}=f(x, u)
$$

in $\mathbf{R}$. Hence, $f$ is a bi-mapping on $\mathbf{R}^{2}$.

However, $f$ is not bilinear, obviously. $f$ is not weakly quasi-linear either. Let

$$
z_{n}=\left(x_{n}, u_{n}\right)=\left(n, \frac{1}{n^{2}}\right)
$$

and

$$
z_{n}^{\prime}=\left(x_{n}^{\prime}, u_{n}^{\prime}\right)=\left(n^{2}, \frac{1}{n^{3}}\right)
$$

where $n \in \mathbf{N}$. Obviously,

$$
f\left(z_{n}\right)=f\left(x_{n}, u_{n}\right)=x_{n}^{2} u_{n}^{2}=n^{2} \cdot \frac{1}{n^{4}} \rightarrow 0
$$

and

$$
f\left(z_{n}^{\prime}\right)=f\left(x_{n}^{\prime}, u_{n}^{\prime}\right)=\left(x_{n}^{\prime}\right)^{2}\left(u_{n}^{\prime}\right)^{2}=n^{4} \cdot \frac{1}{n^{6}} \rightarrow 0 .
$$

But

$$
\begin{gathered}
f\left(z_{n}+z_{n}^{\prime}\right)=f\left(\left(x_{n}, u_{n}\right)+\left(x_{n}^{\prime}, u_{n}^{\prime}\right)\right)=f\left(\left(x_{n}+x_{n}^{\prime}\right),\left(u_{n}+u_{n}^{\prime}\right)\right) \\
=\left(x_{n}+x_{n}^{\prime}\right)^{2}\left(u_{n}+u_{n}^{\prime}\right)^{2}=\left(x_{n} u_{n}+x_{n} u_{n}^{\prime}+x_{n}^{\prime} u_{n}+x_{n}^{\prime} u_{n}^{\prime}\right)^{2} \\
=\left(n \cdot \frac{1}{n^{2}}+n \cdot \frac{1}{n^{3}}+n^{2} \cdot \frac{1}{n^{2}}+n^{2} \cdot \frac{1}{n^{3}}\right)^{2} \rightarrow 1 \neq 0
\end{gathered}
$$

so $f$ is not weakly quasi-linear.

Remark 2.6. For $n \geq 2$, define $f: \mathbf{R}^{2} \rightarrow \mathbf{R}$ by $f(x, u)=x^{n} u^{n}$. Then $f$ is a bi-mapping, but not bilinear, not weakly quasi-linear, and even continuous. 
Example 2.7. Define $f: \mathbf{R}^{2} \rightarrow \mathbf{R}$ by $f(x, u)=\sqrt{x^{2}+u^{2}}$. Then $f$ is a bi-mapping, but not bilinear.

In the following, we will give some propositions which are helpful to our knowledge of bi-mappings in further.

As in [12], denote by $w q l(X, Y)$, the family of all weakly quasi-linear mappings from the topological vector space $X$ to the topological vector space $Y$.

Proposition 2.8. Let $X_{1}, X_{2}$ and $Y$ be Hausdorff topological vector spaces and $X_{1}, X_{2}$ finite-dimensional. If $g \in w q l\left(X_{1}, Y\right)$ and $h \in w q l\left(X_{2}, Y\right)$, and $f: X_{1} \times X_{2} \rightarrow Y$ is defined by

$$
f(x, u)=\alpha g(x)+\beta h(u), \forall x \in X_{1}, u \in X_{2}
$$

for some $\alpha, \beta \in \mathbf{R}$, then $f$ is a continuous bi-mapping, but not bilinear for $g$ or $h$ is not linear.

Proof. As in [12], we know $g(0)=0, h(0)=0$ and $g, h$ both are continuous. So it is easy to know $f$ is continuous and a bi-mapping for $g \in w q l\left(X_{1}, Y\right)$ and $h \in w q l\left(X_{2}, Y\right)$.

Proposition 2.9. Let $\left(X_{1},\|\cdot\|_{1}\right)$ and $\left(X_{2},\|\cdot\|_{2}\right)$ be nontrivial paranormed space [9]. Define $f: X_{1} \times X_{2} \rightarrow \mathbf{R}$ by

$$
f(x, u)=\alpha\|x\|_{1}+\beta\|u\|_{2}, \forall x \in X_{1}, u \in X_{2} .
$$

Then $f$ is a bi-mapping but $f$ is not bilinear when $\|\cdot\|_{1} \neq 0$ or $\|\cdot\|_{2} \neq 0$.

Proof. Following the definition of paranorm [9], it is easy to know.

Proposition 2.10. Let $\varphi:[0,+\infty) \rightarrow(0,+\infty)$ and $\psi:[0,+\infty) \rightarrow(0,+\infty)$ be continuous functions such that

$$
\begin{aligned}
& 0<\mu=\inf _{t \geq 0} \varphi(t) \leq \sup _{t \geq 0} \varphi(t)=M<+\infty . \\
& 0<\mu=\inf _{t \geq 0} \psi(t) \leq \sup _{t \geq 0} \psi(t)=M<+\infty .
\end{aligned}
$$

Let $\left(X_{1},\|\cdot\|_{1}\right),\left(X_{2},\|\cdot\|_{2}\right)$ be Fréchet spaces and $Y$ topological vector spaces. If $g \in w q l\left(X_{1}, Y\right)$ and $h \in w q l\left(X_{2}, Y\right)$ are continuous, and $f: X_{1} \times X_{2} \rightarrow Y$ is defined by

$$
f(x, u)=\varphi\left(\|x\|_{1}\right) g(x)+\psi\left(\|u\|_{2}\right) h(u), \forall x \in X_{1}, u \in X_{2},
$$

then $f$ is a continuous bi-mapping, but not bilinear. 
Proof. We know $\|\cdot\|_{1}: X_{1} \rightarrow \mathbf{R}$ is continuous [9]. So $\varphi\left(\|\cdot\|_{1}\right) g(\cdot)$ : $X_{1} \rightarrow Y$ is continuous for continuous mappings $\varphi$ and $g$. As the same, $\psi\left(\|\cdot\|_{2}\right) h(\cdot): X_{2} \rightarrow Y$ is continuous for continuous mappings $\psi$ and $h$. Then $f$ is continuous on $X_{1} \times X_{2}$.

As in [12], $g(0)=h(0)=0$. Since $0<\mu \leq \varphi(t) \leq M<+\infty$ and $0<\mu \leq \psi(t) \leq M<+\infty$ for all $t \geq 0, \varphi\left(\left\|x_{n}\right\|_{1}\right) g\left(x_{n}\right) \rightarrow 0$ if and only if $g\left(x_{n}\right) \rightarrow 0, \psi\left(\left\|u_{n}\right\|_{2}\right) h\left(u_{n}\right) \rightarrow 0$ if and only if $h\left(u_{n}\right) \rightarrow 0$. Thus, (1) and (2) hold for $f$ for $g \in w q l\left(X_{1}, Y\right), h \in w q l\left(X_{2}, Y\right)$.

Also, for $g(0)=h(0)=0, g \in w q l\left(X_{1}, Y\right)$ and $h \in w q l\left(X_{2}, Y\right)$, (3) hold for $f$ since $f, g, h$ are continuous and $\varphi, \psi$ are continous at 0 . Thus, $f$ is a bi-mapping.

Proposition 2.11. Let $X_{1}, X_{2}$ and $Y$ be metric linear spaces and $f$ : $X_{1} \times X_{2} \rightarrow Y$ a bi-mapping. If $f$ satisfies

$$
\begin{aligned}
& f\left(x_{n}, 0\right) \rightarrow f(x, 0) \Longrightarrow f\left(x_{n}-x, 0\right) \rightarrow 0, \\
& f\left(0, u_{n}\right) \rightarrow f(0, u) \Longrightarrow f\left(0, u_{n}-u\right) \rightarrow 0,
\end{aligned}
$$

and for each $x \in X$ and $u \in U, f(\cdot, u): X_{1} \rightarrow Y, f(x, \cdot): X_{2} \rightarrow Y$ are continuous, then $f$ is continuous.

Proof. If $x_{n} \rightarrow x$ in $X_{1}$ and $u_{n} \rightarrow u$ in $X_{2}$, then $f\left(x_{n}, 0\right) \rightarrow f(x, 0)$ and $f\left(0, u_{n}\right) \rightarrow f(0, u)$ so $f\left(x_{n}-x, 0\right) \rightarrow 0, f\left(0, u_{n}-u\right) \rightarrow 0$ and then $f\left(x_{n}, u_{n}\right) \rightarrow f(x, u)$ since $f$ is a bi-mapping. Thus, $f$ is continuous.

Proposition 2.12. Let $X_{1}, X_{2}$ and $Y$ be topological vector spaces and $f$ a mapping from $X_{1} \times X_{2}$ to $Y$. If $f(\cdot, 0) \in w q l\left(X_{1}, Y\right), f(0, \cdot) \in w q l\left(X_{2}, Y\right)$ and $f$ is continuous, then $f$ is a bi-mapping from $X_{1} \times X_{2}$ to $Y$.

Proof. It is easy to know (1) and (2) hold for $f$ since $f(\cdot, 0) \in w q l\left(X_{1}, Y\right)$, $f(0, \cdot) \in w q l\left(X_{2}, Y\right)$.

If $x_{n} \rightarrow x$ in $X_{1}$ and $u_{n} \rightarrow u$ in $X_{2}$, then $f\left(x_{n}, u_{n}\right) \rightarrow f(x, u), f\left(x_{n}, 0\right) \rightarrow$ $f(x, 0)$ and $f\left(0, u_{n}\right) \rightarrow f(0, u)$ since $f$ is continuous. So $f\left(x_{n}-x, 0\right) \rightarrow$ $0, f\left(0, u_{n}-u\right) \rightarrow 0$ since $f(\cdot, 0) \in w q l\left(X_{1}, Y\right), f(0, \cdot) \in w q l\left(X_{2}, Y\right)$.

Similarly, we can define multi-mappings on topological vector spaces and obtain the multi-mappings version of closed graph theorem on product spaces as follows.

Definition 2.13. Let $X_{1}, X_{2}, \cdots, X_{m}$ and $Y$ be topological vector spaces. A mapping

$$
f: X_{1} \times X_{2} \times \cdots \times X_{m} \rightarrow Y
$$


is said to be a multi-mapping if for each $x^{i}, x_{n}^{i}, u_{n}^{i} \in X_{i}$ with $i=1,2, \cdots, m$ and $n \in \mathbf{N}$ the following (1), (2) and (3) hold:

(1) if $f\left(0, \cdots, 0, x_{n}^{i}, 0, \cdots, 0\right) \rightarrow 0$ and $f\left(0, \cdots, 0, u_{n}^{i}, 0, \cdots, 0\right) \rightarrow 0$, then

$$
f\left(0, \cdots, 0, x_{n}^{i}+u_{n}^{i}, 0, \cdots, 0\right) \rightarrow 0
$$

where $i=1,2, \cdots, m$;

(2) if $f\left(0, \cdots, 0, x_{n}^{i}-x^{i}, 0, \cdots, 0\right) \rightarrow 0$ and $t_{n} \rightarrow t$ in the scalar field $\mathbf{K}$, then

$$
f\left(0, \cdots, 0, t_{n} x_{n}^{i}-t x^{i}, 0, \cdots, 0\right) \rightarrow 0
$$

where $i=1,2, \cdots, m$;

(3) if $x_{n}^{i} \rightarrow x^{i}, i=1,2, \cdots, m$, then

$$
f\left(x_{n}^{1}, x_{n}^{2}, \cdots, x_{n}^{m}\right) \rightarrow f\left(x^{1}, x^{2}, \cdots, x^{m}\right)
$$

if and only if

$$
f\left(0, \cdots, 0, x_{n}^{i}-x^{i}, 0, \cdots, 0\right) \rightarrow 0 \text { for all } i=1,2, \cdots, m
$$

Theorem 2.14. Let $X_{1}, X_{2}, \cdots, X_{m}$ and $Y$ be Fréchet spaces. If

$$
f: X_{1} \times X_{2} \times \cdots \times X_{m} \rightarrow Y
$$

is a multi-mapping with closed graph, then $f$ is continuous.

Remark 2.15. It is similar to Remark 2.3, we know Fernandez's closed graph theorem on product spaces for multilinear mappings in [4] is just a special case of Theorem 2.14.

Acknowledgement. The research is supported by the National Natural Science Foundation of China (Grant No. 11126165) and the Seed Foundation of Tianjin University (Grant No. 60302051). The authors wish to thank the editor and referees. 


\section{References}

[1] S. Banach, Theorie des Operations Lineaires, Warszawa, (1932).

[2] V. Brattka and G. Gherardi, Effective choice and boundedness principles in computable analysis, Bull. Symb. Log. 17, No. 1, pp. 73-117, (2011).

[3] P. J. Cohen, A counterexample to the closed graph theorem for bilinear maps, J. Func. Anal. 16, No. 2, pp. 235-240, (1974).

[4] C. S. Fernandez, Research notes the closed graph theorem for multilinear mappings, Internat. J. Math. Math. Sci. 19, No. 2, pp. 407-408, (1996).

[5] J. C. Ferrando and L. M. S. Ruiz, On C-Suslin spaces, Math. Nachr. 288, No. 8-9, pp. 898-904, (2015).

[6] T. Guo, On some basic theorems of continuous module homomorphisms between random normed modules, J. Funct. Space Appl. (2013), Article Number: 989102.

[7] M. D. Mabula and S. Cobzas, Zabrejko's lemma and the fundamental principles of functional analysis in the asymmetric case, Topology Appl. 184, No. 4, pp. 1-15, (2015).

[8] M. Saheli, A. Hasankhani and A. Nazari, Some properties of fuzzy norm of linear operators, Iran. J. Fuzzy Syst. 11, No. 2, 121-139, (2014).

[9] A. Wilansky, Modern Methods in Topological Vector Spaces, McGrawHill, New York, (1978).

[10] M. Wojtowicz and W. Sieg, P-spaces and an unconditional closed graph theorem, Rev. Real Acad. Cienc. Exactas Fis. Nat. Ser. A-Mat. 104, No. 1, pp. 13-18, (2010).

[11] S. Zhong and R. Li, Continuity of mappings between Fréchet spaces, J. Math. Anal. Appl. 311, No. 2, pp. 736-743, (2005).

[12] S. Zhong, R. Li and S. Y. Won, An improvement of a recent closed graph theorem, Topology Appl. 155, No. 15, pp. 1726-1729, (2008). 
S. Zhong

Department of Mathematics,

Tianjin University,

Tianjin 300072,

Tianjin,

China

e-mail : shuhuizhong@126.com

and

G. Zhao

Weixian Middle School,

Xingtai 054700,

Hebei,

China

e-mail : zhao6158289@163.com 